\title{
AUTOGENOUS LABIAL MUCOUS MEMBRANE AND BANKED SCLERAL PATCH GRAFTING FOR EXPOSED RETINAL EXPLANTS
}

\author{
J. R. MURDOCH, R. SAMPATH, M. J. LAVIN and B. LEATHERBARROW \\ Manchester
}

\begin{abstract}
SUMMARY
Following retinal detachment surgery, exposure of the scleral explant is uncommon and usually requires its removal. In patients with a history of multiple retinal detachment procedures or continuing vitreoretinal traction removal of an explant can lead to retinal redetachment. In such patients it is preferable to cover and retain an exposed explant rather than remove it. Various techniques have been described to cover exposed explants. If the exposed area is small a conjunctivoplasty or conjunctival graft is usually adequate. The use of banked scleral patch grafts has been described in the management of exposed orbital implants. We describe the use of this technique in combination with a labial mucous membrane graft in four patients with an exposed silicone retinal explant. With a minimum follow-up period of 11 months the explant remains covered in all patients.
\end{abstract}

The majority of retinal detachment operations in current practice involve the use of scleral explants. These are usually made of silicone and are sutured to the sclera using non-absorbable suture material. They are then covered with anterior Tenon's fascia and conjunctiva. The explants usually remain buried beneath these layers, but occasionally they may become exposed (Fig. 1) due to atrophy or breakdown of the overlying tissues, or conjunctival wound dehiscence. Conjunctival wound dehiscence is often associated with areas of episcleral and scleral vascular closure which may occur after cryotherapy. Conjunctival dehiscence may also be associated with a prominent or mobile scleral explant. Exposed explants predispose to infection and discomfort and in most cases should be removed. This can, however, sometimes lead to redetachment of the retina.

Correspondence to: Mr John R. Murdoch, FRCS, FRCOphth, Stobhill NHS Trust, 133 Balornock Road, Glasgow G21 3UW, UK.
Retinal redetachment rate following explant removal has been variously reported as between $4 \%$ and $47 \%{ }^{1-4}$ Patients with a history of multiple retinal detachment procedures, those with continuing vitreoretinal traction, patients with proliferative vitreoretinopathy, or aphakic patients with multiple small post-oral breaks are especially at increased risk

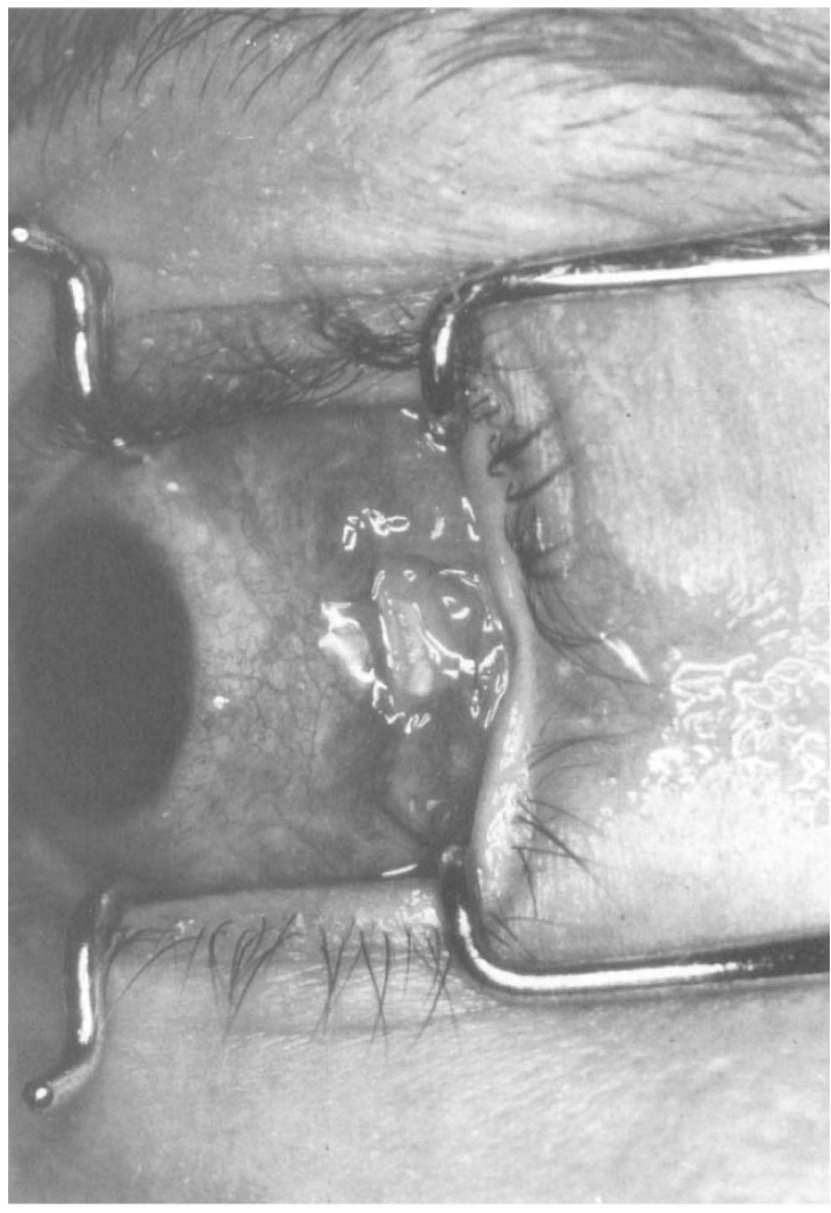

Fig 1. Exposed silicone retinal explant.

Eye (1997) 11, 43-46 (C) 1997 Royal College of Ophthalmologists 
of retinal redetachment following explant removal. In these groups of patients it is preferable to cover the buckle rather than to remove it, provided there is no evidence of infection.

There are various techniques which can be used to cover an exposed retinal explant. If the exposure is small a conjunctivoplasty or conjunctival graft from the same or fellow eye is usually adequate, but this will not work if the exposure is large. Autogenous fascia lata has been used to cover exposed retinal explants ${ }^{5}$ but this technique is time consuming and carries a risk of morbidity. The use of a banked scleral graft has been described in the management of exposed orbital implant ${ }^{6-9}$ and exposed scleral explants after retinal detachment surgery. ${ }^{10} \mathrm{We}$ describe the use of this technique in combination with a labial mucous membrane graft in four patients with an exposed silicone retinal explant.

\section{Surgical Procedure}

\section{METHODS}

The same surgical technique was used in all four patients. None of the patients had clinical evidence of infection (significant conjunctival hyperaemia or purulent conjunctival discharge) and all had culture-negative conjunctival swabs. All patients were

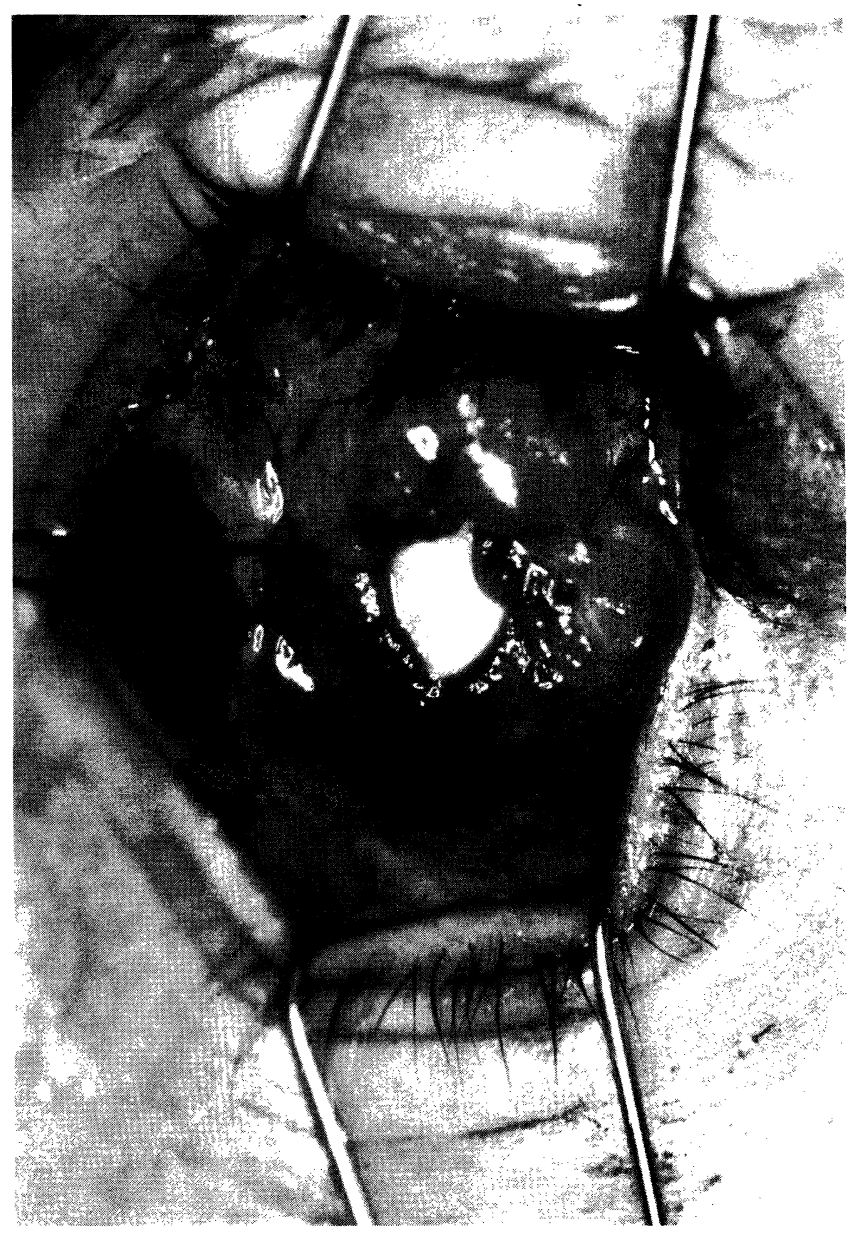

Fig. 2. Retinal explant covered with scleral patch graft. treated with pre-operative topical chloramphenicol. The procedure was performed under general anaesthesia. The patients were prepared and draped in a standard sterile ophthalmic fashion. A 4.0 silk episcleral traction suture was placed at the limbus adjacent to the conjunctival dehiscence and the globe rotated to provide maximum exposure. The conjunctival dehiscence was explored and its dimensions measured. A slightly over-sized donor scleral patch graft was fashioned. This was folded and inserted through the conjunctival wound and carefully positioned to lie over the exposed retinal explant. The scleral patch graft was unfolded underneath the conjunctiva to lie flat (Fig. 2).

Next the lower lip was injected with $5 \mathrm{ml}$ of $0.5 \%$ Marcaine with 1:200000 units of adrenaline. This was followed by an injection of $10 \mathrm{ml}$ of normal saline. Babcock's clamps were applied to the lip and placed on traction. A template of the area of conjunctival dehiscence was made using a piece of Steridrape and applied to the mucosa of the lower lip. The full-thickness mucous membrane graft was harvested using a no. 15 Bard Parker blade. Haemostasis was obtained using bipolar cautery and a swab moistened with 1:1000 units of adrenaline. The Babcock's clamps were then removed.

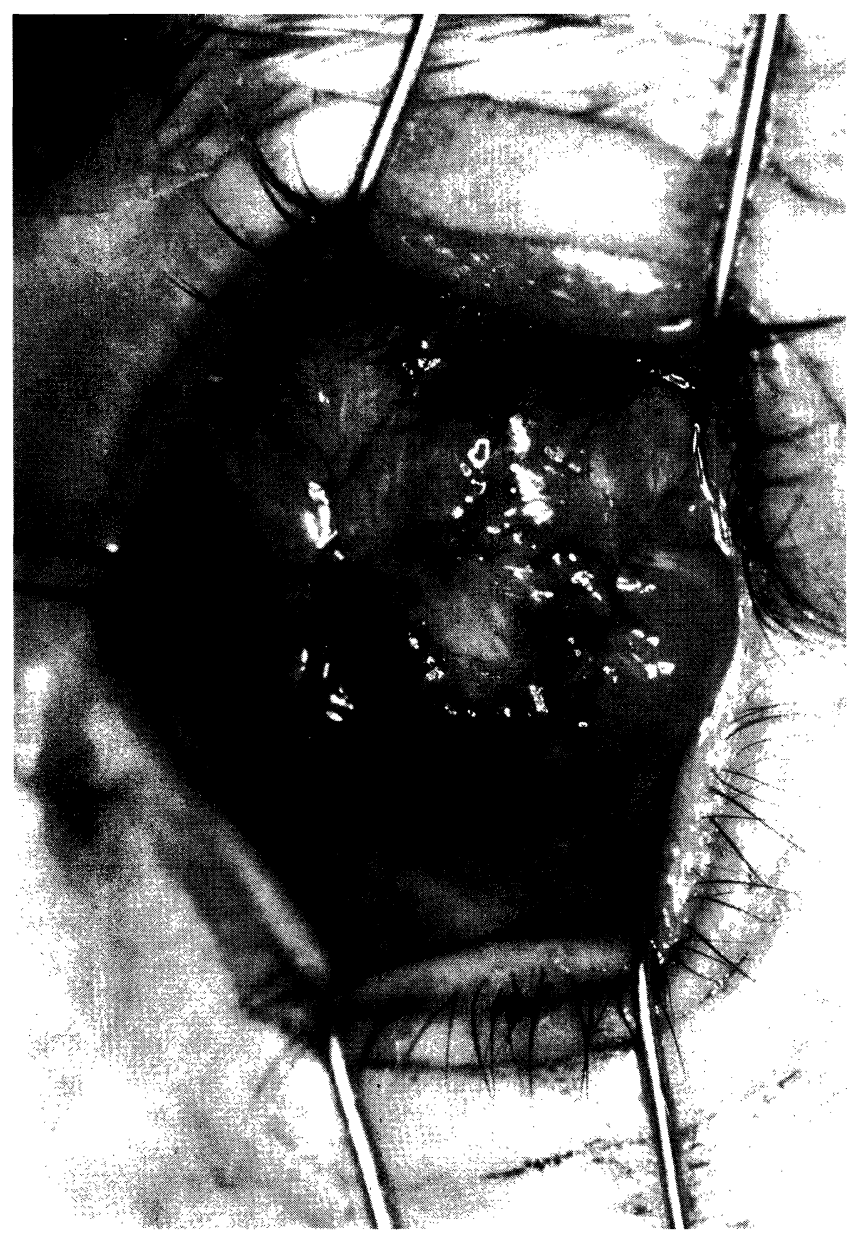

Fig. 3. Labial mucous membrane graft sutured in place. 


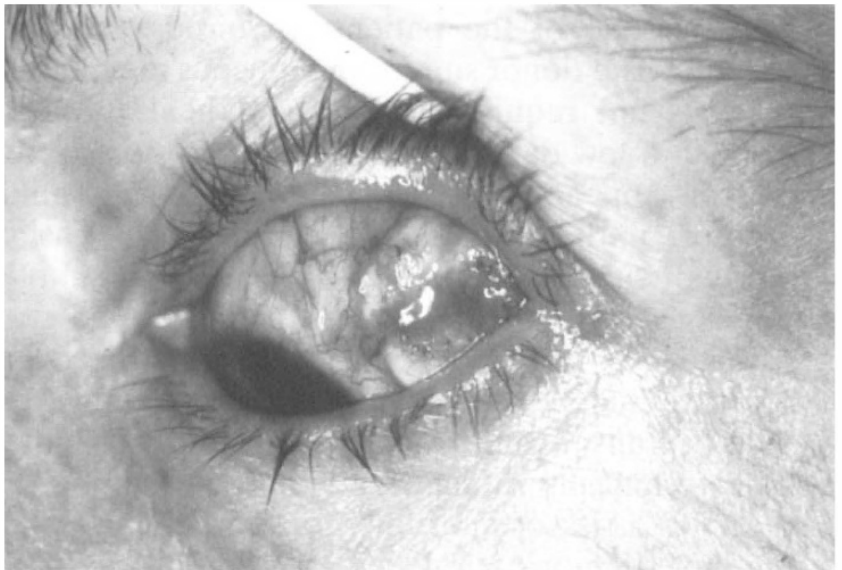

Fig. 4. Appearance of retinal explant covered with scleral patch graft and labial mucous membrane graft 4 weeks after surgery.

The mucous membrane graft was carefully thinned using Westcott's scissors and the graft was placed over the conjunctival hole. The graft was then carefully sutured in place using interrupted 7.0 vicryl sutures (Fig. 3). Topical antibiotic ointment and a firm pressure dressing were applied.

Post-operatively the pressure dressing remained in place for 7 days. Corsodyl mouth washes were used as required and chloramphenicol eye drops were instilled four times daily for 6 weeks. The appearance of a retinal explant covered with scleral patch graft and labial mucous membrane graft 4 weeks after surgery is shown in Fig. 4.

\section{Case 1}

\section{CASE REPORTS}

A 74-year-old woman who had previously undergone bilateral intracapsular cataract surgery complicated by bilateral cystic bleb formation and an inoperable right retinal detachment developed a leaking left conjunctival bleb. This was excised and conjunctiva was sutured over the scleral fistula track. Following the procedure the leak persisted for 1 week before spontaneously resolving. Three years later she developed a left temporal retinal detachment which was repaired conventionally using cryotherapy and a 277 explant with a 240 encircling band. Eight months after this operation she developed a superotemporal conjunctival sinus and exposure of the retinal explant. She was referred for a donor scleral patch graft and mucous membrane graft. This procedure successfully covered the exposed explant. Five months later she developed a further left retinal detachment which was repaired via a two-port nasal vitrectomy using endolaser to avoid interfering with her scleral patch graft. Sixteen months after this operation her corrected visual acuity is 6/18. Her retina is flat and the explant remains covered.
Case 2

A 27-year-old highly myopic man with a strong family history of retinal detachment and possibly Stickler's syndrome who had previously undergone conventional bilateral retinal detachment surgery developed an exposed retinal explant in the superotemporal quadrant of the left eye. In view of the high risk of retinal redetachment it was thought desirable to cover the explant rather than remove it. An initial attempt to cover the explant with conjunctival flaps was unsuccessful and the patient was referred for donor scleral patch graft and labial mucous membrane graft. Seventeen months after the procedure the explant again became exposed temporally and a further donor scleral patch graft and labial mucous membrane graft was inserted. Nineteen months postoperatively the explant remains covered and the retina attached.

\section{Case 3}

A 54-year-old man who had previously undergone a right cataract extraction complicated by vitreous loss developed a right superior bullous rhegmatogenous retinal detachment with a posterior retinal tear adjacent to a patch of lattice degeneration. He underwent a right vitrectomy with $\mathrm{SF}_{6}$ gas, cryotherapy and placement of a 279 explant. One month post-operatively he developed a further right rhegmatogenous retinal detachment associated with proliferative vitreoretinopathy and a small nasal post-oral tear. He underwent a further vitrectomy with retinotomy, endolaser and $\mathrm{C}_{3} \mathrm{~F}_{8}$ gas. His retina remained flat with a corrected visual acuity of $6 / 9$. Five months after surgery he developed several small temporal conjunctival sinuses which tracked back to the scleral buckle and caused discomfort and recurrent conjunctivitis. Rather than risk redetachment by removing the buckle his conjunctival dehiscence was explored under general anaesthesia and was found to measure $5 \mathrm{~mm} \times 6 \mathrm{~mm}$. The exposed explant was covered using a donor scleral patch graft and labial mucous membrane graft. Eleven months post-operatively the explant remains covered and the retina attached.

\section{Case 4}

A 32-year-old diabetic woman who had end-stage proliferative retinopathy in her right eye with a visual acuity of no perception of light, developed a total combined tractional rhegmatogenous retinal detachment in her left eye. She underwent a left vitrectomy with silicone oil and an inferior $200^{\circ} 277$ explant. Post-operatively her retina remained securely attached and her visual acuity improved to $6 / 60$. Eighteen months following her surgery the explant became exposed temporally, the size of the conjunctival dehiscence measuring $12 \mathrm{~mm} \times 5 \mathrm{~mm}$. As 
there was continuing vitreoretinal traction in this patient's only eye it was felt desirable to cover the explant rather than remove it. The exposed explant was covered using a donor scleral patch graft and labial mucous membrane graft. Fourteen months post-operatively the explant remains covered and the retina attached.

\section{DISCUSSION}

We believe that in patients with exposed retinal explants in whom removal of the explant is undesirable, covering the explant with a banked scleral patch graft and autogenous labial mucous membrane graft is a straightforward successful technique. Although there is a theoretical risk of transmission of infection from donor material, this risk is minimised by careful selection of donors and preparation of the material. Sclera was obtained from our local eye bank. All donors are screened for hepatitis B, hepatitis $\mathrm{C}$ and HIV infection. Donor material is soaked in $5 \%$ povidone iodine for 30 minutes before storage in $100 \%$ alcohol for a minimum period of 28 days. Prior to its use the donor sclera is placed in gentamicin solution. We are not aware of any reports of patients developing infectious disorders as a result of the use of donor sclera.

In a previous report of sclera being used to cover an exposed retinal explant, Watzke ${ }^{10}$ sutured the sclera to the conjunctival margins. The scleral graft was left bare and allowed to 'conjunctivalise' spontaneously over a period of approximately 4 weeks. In patients who have had multiple retinal reattachment procedures, including cryotherapy, the conjunctiva may be unhealthy and this process may be slow. Exposed sclera may cause discomfort for the patient. For these reasons we have covered the sclera with a labial mucous membrane graft. There is no need to suture conjunctiva to sclera. The mucous membrane graft is more comfortable for the patient and ensures that the post-operative recovery is more rapid without the need for frequent follow-up appointments. In our series of four patients, all mucous membrane grafts have survived and exposed silicone retinal explants have remained with a minimum follow-up time of 11 months. It should be noted that one of the patients required a second procedure. The donor site for the mucous membrane graft does not require suturing and heals spontaneously in a few days. The donor site, in spite of its initial raw appearance, causes the patient only mild discomfort until it heals. The discomfort is easily controlled with Corsadyl mouth washes and simple oral analgesics.

Autogenous fascia lata has been used to cover exposed retinal explants ${ }^{5}$ and has the advantage of no risk of transmissible infection, but this procedure can be technically difficult and time consuming. It also carries a risk of morbidity for the patient.

We have demonstrated that covering an exposed retinal explant with a banked scleral patch graft and autogenous labial mucous membrane graft is a straightforward successful technique. We recommend this procedure for patients in whom it is important to retain an exposed silicone explant.

Key words: Mucous membrane graft, Retinal detachment surgery, Retinal explant, Scleral patch graft.

\section{REFERENCES}

1. Deutsch J, Aggarwal RK, Eagling EM. Removal of scleral explants: a 10 year retrospective study. Eye 1992;6:570-3.

2. Lindsey P, Pierce L, Welch R. Removal of scleral explant elements. Arch Ophthalmol 1983;101:570-3.

3. Hilton G, Wallyn R. The removal of scleral buckles. Arch Ophthalmol 1978;96:2061-3.

4. Schwarz PI, Pruett RC. Factors influencing retinal redetachment after removal of explant elements. Arch Ophthalmol 1977;95:804-7.

5. Dressner SC, Boyer DS, Feinfield RE. Autogenous fascial grafts for exposed retinal buckles. Arch Ophthalmol 1991;109:288-9.

6. Helveston EM. Human bank scleral patch for repair of exposed or extruded orbital implants. Arch Ophthalmol 1969;82:83-6.

7. Soll DB. The use of sclera in surgical management of extruding implants. Ophthalmology 1978;85:863-8.

8. Fountain JA, Helveston EM. A long-term follow-up study of scleral grafting for exposed or extruded orbital implants. Am J Ophthalmol 1982;93:52-6.

9. Goldberg MF. A simplified scleral graft technique for covering an exposed orbital implant. Ophthalmic Surg 1988;19:206-11.

10. Watzke RC. Scleral patch graft for exposed episcleral implants. Arch Ophthalmol 1984;102:114-5. 